\title{
Evolución temporal de la incidencia por incapacidad temporal de origen común según tramos de duración (2009-2018)
}

Time series of non-work related sickness absence incidence by subgroups of duration (2009-2018)

\author{
Mónica Ballesteros Polo ${ }^{1}$ \\ Maite Sampere Valero' \\ Juan Carlos López López ${ }^{1}$ \\ Rosa Sacristán Nieto' \\ Ana Ruiz Julián ${ }^{1}$ \\ Sara Alguacil García ${ }^{1}$ \\ Elisabeth Santos Ávila' \\ Silvia García Rubia' \\ Bibiana López Costa ${ }^{\top}$ \\ José Miguel Martínez Martínez \\ ${ }^{1}$ MC Mutual, Barcelona, España. \\ ${ }^{2}$ Departamento de Estadística e Investigación Operativa, Universitat Politècnica de Catalunya, Barce- \\ lona, España. \\ ${ }^{3}$ Grupo de Investigación en Salud Pública, Universidad de Alicante, Alicante, España.
}

Fechas · Dates

Recibido: 2019.07.26

Aceptado: 2019.12.27

Publicado: 2020.04.15
Correspondencia · Corresponding Author

Mónica Ballesteros Polo

Departamento de Investigación y Análisis de Prestaciones, MC Mutual.

Provenza, 321. 08037 Barcelona

mballesteros@mc-mutual.com 


\section{Resumen}

Objetivo: Describir la evolución temporal (2009-2018) de la incidencia de la incapacidad temporal por contingencia común (ITCC) según tramos de duración en España.

Métodos: Se consideraron casos de ITCC de la población afiliada de una mutua laboral. Se obtuvo la incidencia global de la incapacidad temporal por ITCC y por tramos de duración (1-3 días, 4-15 días, 16-30 días, 31-90 días y >90 días). Se realizó un estudio ecológico de series temporales considerando un punto de inflexión en 2013. Se calculó el porcentaje de cambio anual e intervalo de confianza del 95\%. Los análisis se estratificaron por edad y sexo.

Resultados: La incidencia global pasó de 35,3 casos por cada 100 trabajadores-año en 2009 a 25,2 en 2013. A partir del 2014 se observa un ascenso sostenido de la incidencia de ITCC, finalizando el 2018 con 34,1 casos por cada 100 trabajadores-año. La incidencia global está determinada fundamentalmente por procesos menores a 16 días en población joven. El descenso en 2009-2013 se produjo en todos los tramos de duración, en especial en 4 a $15(P C A=-11,2 ;$ IC 95\%=(-14,1 a -8,2)) y más de 90 días $(P C A=-9,4$; IC 95\%=(-15,5 a $-2,8))$, principalmente en jóvenes. El ascenso en 2013-2018 se observó en todos los tramos, siendo el incremento más significativo en tramos de 1-3 días (trabajadores de menor edad: PCA=18,9; IC 95\%=(14,8 a 23,2)) y en más de 90 días (principalmente en mayores). La evolución de la ITCC presentó un patrón similar en ambos sexos.

Conclusiones: La descripción temporal de la incidencia de ITCC por tramos de duración ofrece una información detallada de la incapacidad temporal. Estos resultados son útiles para profesionales de la prevención y gestión de la ITCC.

Palabras clave: Ausencia por enfermedad; Incidencia; Lugar de trabajo; Estudios de series temporales

\section{Abstract}

Objective: We describe time trend incidence (2009-2018) of non-work related Sickness Absence (SA) segmented by duration of episodes in Spain.

Methods: We used SA cases from a health insurance company ("mutua") in Spain. Overall non-work related SA incidence and incidence by duration of episodes (1-3 days, 4-15 days, 16-30 days, 31-90 days and $>90$ days) were obtained. A time series ecological study was carried out with an inflection point in 2013 . The annual percentage of change and $95 \%$ confidence interval were obtained. The analyses were stratified by age and sex.

Results: Overall incidence went from 35.3 cases per 100 workers-years in 2009 to 25.2 in 2013. From 2014, there is a sustained rise in the incidence of ITCC, ending 2018 with 34.1 cases per 100 workers-years. The overall incidence is determined mainly by processes less than 16 days in young population. The decrease in 2009-2013 occurred in all the duration segments, especially in 4 to $15(\mathrm{APC}=-11,2 ; 95 \% \mathrm{Cl}=(-14,1$ a $-8,2))$ and more than 90 days (APC $=-9,4 ; 95 \% \mathrm{Cl}=(-15,5$ a $-2,8)$ ), mainly in young people. The rise in 2013-2018 was observed in all the segments, with the largest significant increase in sections of 1-3 days (younger workers: $\mathrm{APC}=18,9 ; 95 \% \mathrm{Cl}=(14,8$ a 23,2)) and in more than 90 days (mainly in older ones). Time trend of SA showed similar pattern in both sexes. 
Conclusions: Time trend analysis of SA incidence by duration segments offers a detailed information of SA. These results are useful for professionals in the prevention and management of SA.

Key words: Sick leave; Incidence; Workplace; Time series studies

\section{Introducción}

Los cambios producidos en el contexto laboral y socioeconómico en España han impactado en la evolución del absentismo laboral por incapacidad temporal por contingencia común (ITCC) durante la última década. Estos cambios hacen necesario realizar un estudio sistemático de la evolución temporal. Los informes del Ministerio de Trabajo, Migraciones y Seguridad Social (MITRAMISS) ofrecen valores elevados de la incidencia por contingencia común, con máximos en 2009, seguido de un descenso sostenido hasta 2013, año a partir del cual se inicia una fase ascendente mantenida en la actualidad ${ }^{(1)}$.

De forma paralela, el gasto de la Seguridad Social en prestaciones económicas por ITCC ha presentado un repunte a partir del 2013, alcanzando en el 2018 un coste que ascendió a 7.498.54 Millones de Euros ${ }^{(2)}$. Esto supone un importante reto para las mutuas colaboradoras con la Seguridad Social (en adelante mutuas) y entidades gestoras, exigiendo nuevos planteamientos para el abordaje de la ITCC.

En un reciente estudio, se señalaba que el análisis de la tasa de incidencia de los procesos segmentada por tramos de duración (corta, media y larga), permite gestionar mejor la ITCC que si nos basamos únicamente en incidencias globales sin segmentar ${ }^{(3)}$. Por otro lado, muchos análisis resumen el comportamiento de la serie temporal mediante el porcentaje de cambio anual (PCA) de todo el periodo estudiado. Este enfoque considera que la incidencia aumenta o disminuye de forma constante durante el periodo de estudio. Sin embargo, esta asunción puede no darse con indicadores que presentan cambios o inflexiones destacables en el tiempo. En este caso, se puede obtener un estudio más preciso, mediante el PCA dentro de periodos en los que se pueda asumir está asunción(4). Por lo tanto, es útil evaluar si la evolución temporal de la incidencia por ITCC varía en función de la duración de los procesos teniendo en cuenta posibles puntos de inflexión en la tendencia temporal. También se deben considerar características sociodemográficas como la edad y el sexo, que han demostrado su impacto en el comportamiento de los indicadores ${ }^{(5-9)}$. Esto permitiría avanzar en el conocimiento acerca de la tipología de las bajas (cortas, medias, largas) que predominan y su evolución en el tiempo, que a su vez facilitaría la gestión del absentismo laboral por ITCC.

El objetivo de este estudio es describir la evolución temporal de la incidencia de la ITCC según tramos de duración desde el 2009 hasta el 2018 en España por edad y sexo. 


\section{Métodos}

\section{Diseño de estudio}

Diseño ecológico de series temporales.

\section{Población, lugar y tiempo}

Se consideraron casos de ITCC de la población afiliada por cuenta ajena de una mutua colaboradora con la seguridad social desde el año 2009 al 2018. Esta mutua tiene cobertura nacional, con 1.401.953 trabajadores protegidos en 2018, de los cuales 952.488 tenían la gestión de la contingencia común por cuenta ajena. La mutua dispone de más de 100 centros propios en todo el territorio nacional. También tiene suscritos acuerdos de colaboración con otras mutuas del sector que amplían la red de centros para una mayor cobertura.

\section{Variables}

Las variables dependientes son el número de casos de ITCC global y el número de casos de ITCC según tramos de duración: 1-3 días, 4-15 días, 16-30 días, 31-90 días y > 90 días Los tramos se crearon según los días transcurridos desde el inicio de la baja médica hasta el alta. La variable explicativa será el año que tomará 10 valores correspondientes a los años de 2009 a 2018. Como variables de estratificación se consideró el sexo y la edad (<25, 25-34, 35-44, 45-54 y 55-64 años).

\section{Análisis estadístico}

Se calculó la tasa de incidencia de la ITCC por 100 trabajadores-año (TI) para cada uno de los años de estudio. La TI se obtuvo mediante el cociente entre el número de casos de ITCC que se inician en el periodo de estudio y el promedio anual de trabajadores, calculado como la media entre el número de trabajadores con contrato activo el último día de cada mes. La TI se obtuvo de forma global y segmentada por tramos de duración.

Se describió la evolución temporal de la TI mediante gráficos de series temporales. Se exploró la existencia de inflexiones en la evolución mediante la inspección del gráfico y la realización de una regresión LOESS (Local regression)(10). Para determinar el valor del parámetro de suavizado se usó validación-cruzada(11) a partir del paquete fANCOVA de $\mathrm{R}^{(12)}$. A partir de los resultados obtenidos se definieron dos períodos en función de una inflexión en la tendencia en 2013: 2009-2013 y 20132018. Seguidamente se calculó el porcentaje de cambio anual (PCA) e intervalo de confianza al 95\% (IC 95\%) mediante un modelo Poisson con una estimación robusta para el error estándar. El modelo consideró un punto de inflexión en el año 2013. Específicamente, se consideró que $O_{t}$, el número de casos de ITCC en el año 
t $(2009, . . ., 2018)$ para cada segmento de duración, siguió una distribución Poisson con la siguiente media log-lineal ${ }^{(13)}$ :

$$
\log \left(E\left[O_{t}\right]\right)=\beta_{0}+\beta_{1} t+\beta_{2}(t-2013)^{+}+\log \left(P_{t}\right)
$$

donde $P_{t}$ es promedio anual de trabajadores en el año $t$. La expresión $(t-2013)^{+}$es igual a 0 si $t \leq 2013$ e igual a $(t-2013)$ si $t>2013$. El PCA del periodo 2009-2013 se obtendrá como $\left(e^{\beta_{1}}-1\right) \times 100$ y el del periodo $2013-2017$ como $\left(e^{\beta_{1}+\beta_{2}}-1\right)$. El análisis se estratificó también por edad y sexo. Los programas estadísticos utilizados fueron R y RStudio(14-15).

\section{Aspectos éticos}

Los datos han sido tratados de forma agregada y anónima, de tal manera que no permiten la identificación de ninguna persona, de acuerdo al cumplimiento del Reglamento del Parlamento Europeo y del Consejo (UE) 2016/679, también conocido como Reglamento General de Protección de Datos (RGPD) ${ }^{(16)}$.

\section{Resultados}

La distribución (porcentaje) de la población protegida según sexo, edad y tres de los años de estudio $(2009,2013,2018)$, aparece representada en la Figura 1 . El gráfico nos muestra que dicha población es estable en los tres periodos analizados con una mayor representación en el grupo de 35 a 44 años.
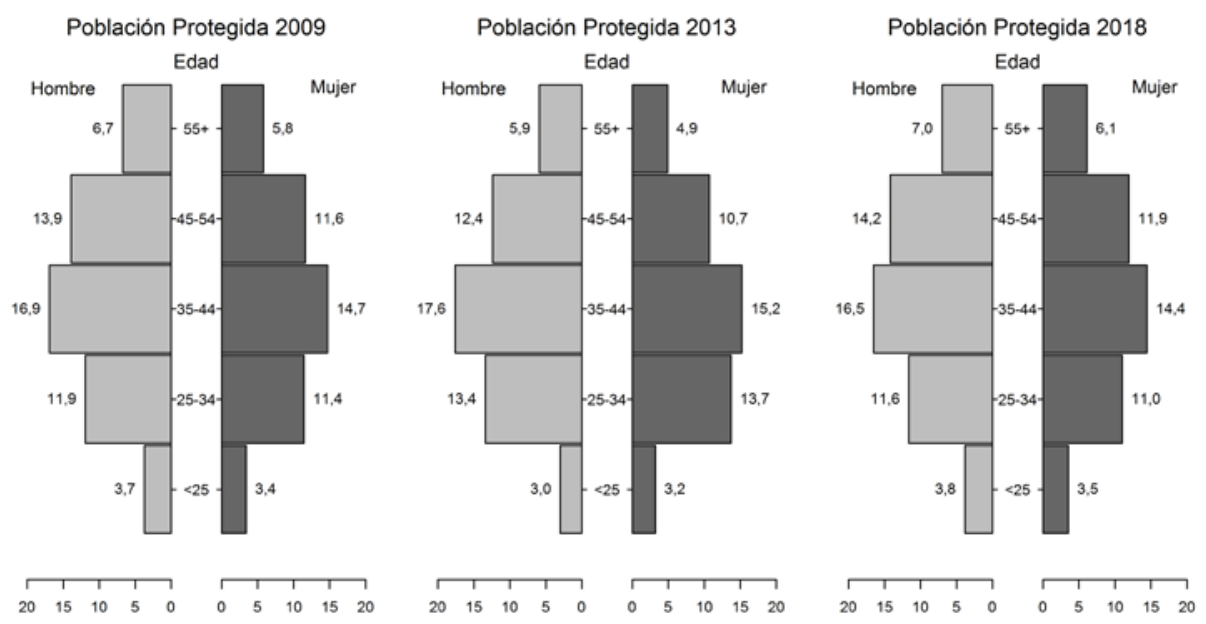

Figura 1: Distribución (porcentaje) de la población protegida según sexo, edad y periodo temporal $(2009,2013,2018)$.

La evolución de los procesos de incapacidad temporal por contingencia común presenta una disminución constante desde 2009 hasta 2013. En 2009 se registraron 248.798 casos de ITCC, alcanzando los 180.283 casos en 2013. Por su 
parte, la tasa de incidencia global disminuyó de 35,3 casos por cada 100 trabajadores-año en 2009 a 25,2 en 2013.

A partir del 2014 se observa un ascenso sostenido de los procesos de ITCC, finalizando el 2018 con más de 324 mil casos y una TI de 34,1 casos por cada 100 trabajadores-año, un 23\% por encima de la reportada en 2013 (Figura 2). En el análisis por tramos de duración, son los procesos con duración inferior a 16 días (1 a 3 días y de 4 a 15 días), los que presentaron un mayor peso respecto a la incidencia global, con valores que se situaron por encima de los otros, alcanzando diferencias entre ellos, como mínimo dos veces superior.

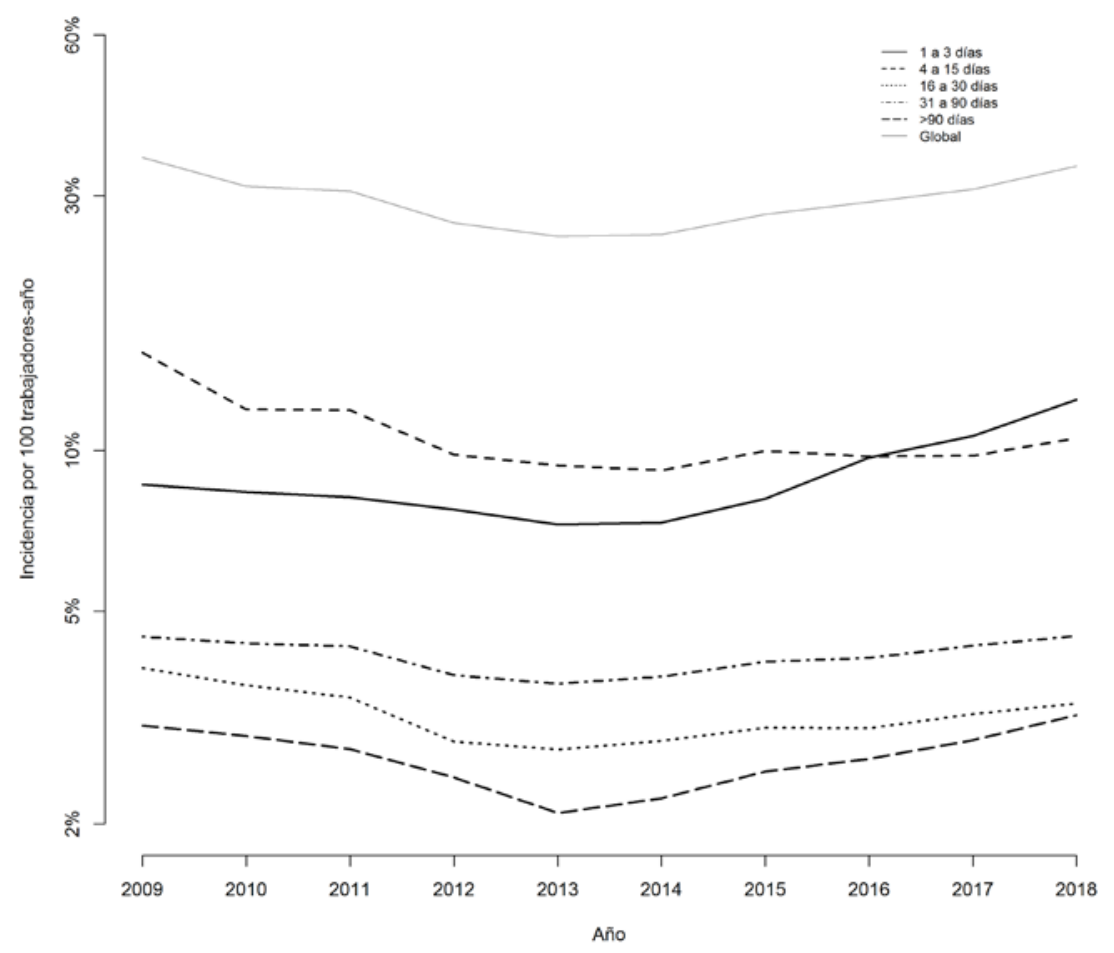

Figura 2: Evolución de la incidencia incapacidad temporal de origen común (ITCC) por tramos de duración (escala logarítmica) durante el período 2009 - 2018.

En la Figura 3 se observa claramente la existencia de un punto de inflexión en el año 2013 en todos los tramos de duración. 

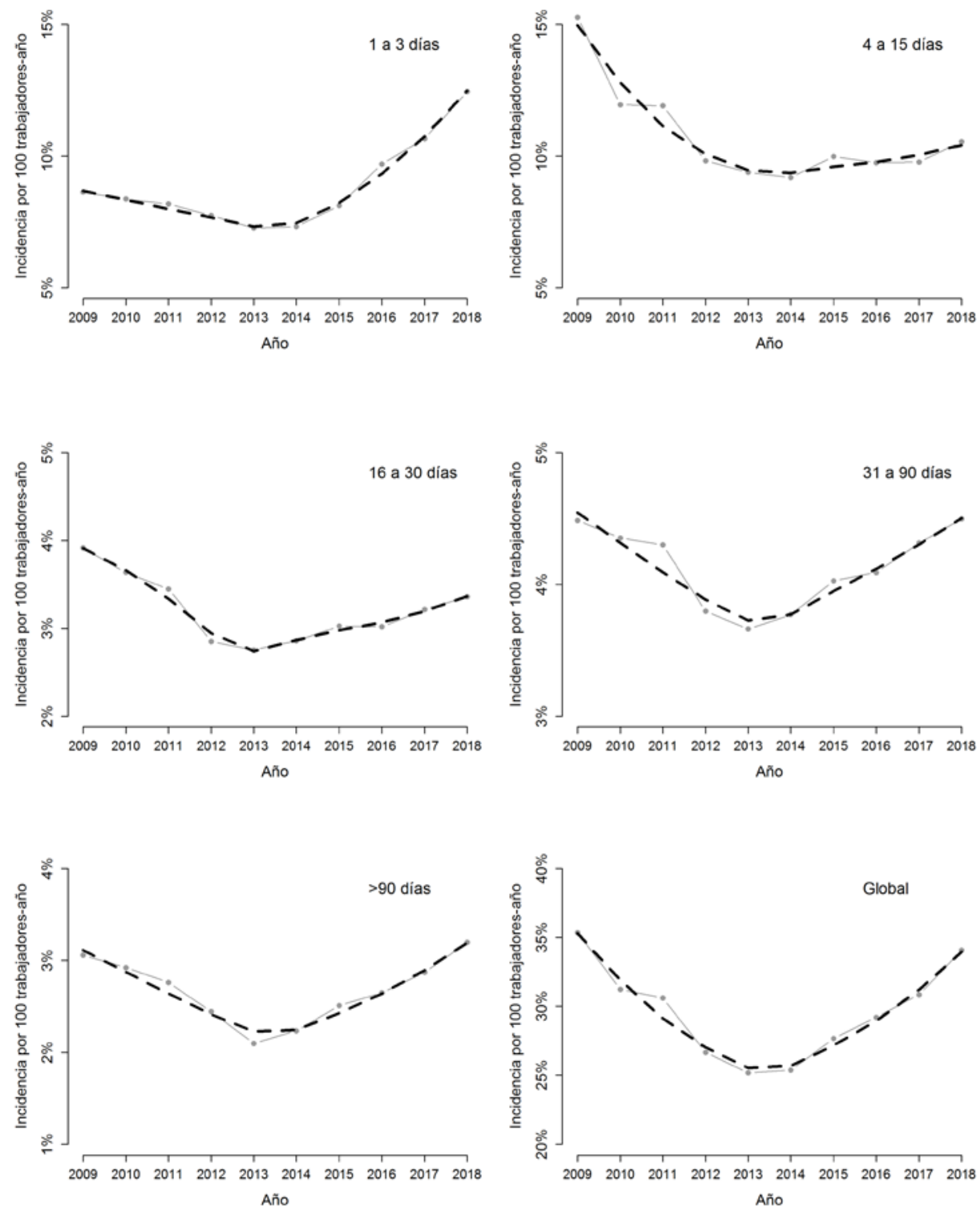

\section{Observado}

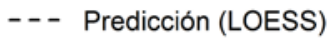

Figura 3: Descripción de la evolución de la incidencia incapacidad temporal de origen común (ITCC) por tramos de duración durante el período 2009 - 2018 según valores observados y predichos mediante regresión LOESS (Local regressionª). 
La caída de la incidencia observada durante el período 2009-2013 afectó de forma significativa a todos los tramos de duración (Tabla 1), siendo más acentuado en los tramos de 4 a 15 días (PCA=-11,2; IC 95\%=(-14,1 a -8,2)) y mayor de 90 días $(P C A=-9,4$; IC 95\%=(-15,5 a -2,8)). Durante el período 2013-2018, el ascenso en la incidencia se produjo en todos los tramos, siendo los tramos de duración extremos los que presentan el incremento de mayor magnitud (PCA en 1 a 3 días= 11,7; IC $95 \%=(6,5$ a 17,2$)$ y PCA en $>90$ días $=7,9 ;$ IC $95 \%=(2,0$ a 14,1$))$.

Al segmentar por edad se observa como la caída de la incidencia durante el período 2009-2013 se dio especialmente en los más jóvenes. Durante el período 20132018 el efecto observado en el tramo de 1 a 3 días se produce principalmente en los menores de 25 años (PCA=18,9; IC 95\%=(14,8 a 23,2)), mientras que en el tramo mayor de 90 días, fue en los grupos de más edad. En este último tramo, se observa que los mayores de 55 años presentaron en 2013 una tasa de incidencia 3,7 veces superior a la de los más jóvenes (4,0 frente a 1,1 respectivamente), mientras que en 2018 la diferencia se multiplicó por $5(6,0$ frente a 1,2) atribuible a un ascenso en la incidencia de los mayores (Tabla 2).

Al analizar los resultados según sexo, la evolución de la TI global y por tramos de duración muestra el mismo patrón que el descrito previamente, con un descenso en 200-2013, seguido de un ascenso en 2013-2018, sin observar diferencias destacables en la evolución entre mujeres y hombres (Tabla 3).

Tabla 1: Incidencia de incapacidad temporal de origen común (ITCC) con sus Intervalo de Confianza del 95\% (IC 95\%) y porcentaje de cambio anual (PCA) por tramos de duración durante los períodos 2009-2013 y 2013-2018.

\begin{tabular}{|c|c|c|c|c|c|c|c|}
\hline & \multicolumn{5}{|r|}{ 2009-2013 } & \multicolumn{2}{|c|}{ 2013-2018 } \\
\hline & $\begin{array}{l}\text { Tasa } \\
2009\end{array}$ & $\begin{array}{l}\text { Tasa } \\
2013\end{array}$ & $\begin{array}{l}\text { Tasa } \\
2018\end{array}$ & PCA & (IC 95\%) & PCA & (IC 95\%) \\
\hline 1 a 3 días & 8,6 & 7,3 & 12,5 & $-6,0$ & $(-11,2 \text { a } 0,5)^{\star \star}$ & 11,7 & $(6,5 \text { a } 17,2)^{\star \star \star}$ \\
\hline 4 a 15 días & 15,3 & 9,4 & 10,6 & $-11,2$ & $(-14,1 a-8,2)^{\star \star}$ & 2,7 & $(0,8 \text { a } 4,7)^{\star \star}$ \\
\hline 16 a 30 días & 3,9 & 2,8 & 3,4 & $-8,9$ & $(-12,6 a-5,0)^{\star \star \star}$ & 4,1 & $(1,2 \text { a } 7,1)^{\star \star}$ \\
\hline 31 a 90 días & 4,5 & 3,7 & 4,5 & $-5,4$ & $(-10,6 \text { a } 0,1)^{\star \star}$ & 4,1 & $(0,1 \text { a } 8,2)^{\star \star}$ \\
\hline > 90 días & 3,1 & 2,1 & 3,2 & $-9,4$ & $(-15,5 a-2,8)^{\star \star}$ & 7,9 & $(2,0 \text { a } 14,1)^{\star \star \star}$ \\
\hline Global & 35,3 & 25,2 & 34,1 & $-8,7$ & $(-11,8 a-5,4)^{\star \star}$ & 6,4 & $(3,8 \text { a } 9,0)^{\star \star}$ \\
\hline
\end{tabular}

${ }^{*} p<0,10 ;{ }^{* *} p<0,05$ 
Tabla 2: Incidencia de incapacidad temporal de origen común (ITCC) con sus Intervalo de Confianza del 95\% (IC 95\%) y porcentaje de cambio anual (PCA) por tramos de duración según edad durante los períodos 2009-2013 y 2013-2018.

\begin{tabular}{|c|c|c|c|c|c|c|c|}
\hline & \multicolumn{5}{|r|}{$2009-2013$} & \multicolumn{2}{|r|}{ 2013-2018 } \\
\hline & $\begin{array}{l}\text { Tasa } \\
2009\end{array}$ & $\begin{array}{l}\text { Tasa } \\
2013\end{array}$ & $\begin{array}{l}\text { Tasa } \\
2018\end{array}$ & PCA & (IC 95\%) & PCA & (IC 95\%) \\
\hline \multicolumn{8}{|l|}{1 a 3 días } \\
\hline$<25$ años & 15,5 & 10,2 & 22,8 & $-13,0$ & $(-15,7 a-10,2)^{\star \star}$ & 18,9 & $(14,8 \text { a } 23,2)^{\star x}$ \\
\hline 25-34 años & 10,9 & 9,8 & 17,3 & $-4,4$ & $(-6,8 \mathrm{a}-2,0)^{\star \star}$ & 12,1 & $(9,6 \text { a 14,7) })^{\star \star}$ \\
\hline 35-44 años & 7,6 & 7,2 & 12,4 & $-2,7$ & $(-5,6 a \text { a } 0,3)^{\star}$ & 11,8 & $(8,8 \text { a } 14,9)^{\star \star}$ \\
\hline 45-54 años & 5,6 & 5,1 & 8,4 & $-4,3$ & $(-10,3$ a 2,2$)$ & 11,0 & $(5,9 \text { a } 16,3)^{\star \star}$ \\
\hline$\geq 55$ años & 3,9 & 4,2 & 6,4 & 0,7 & $(-6,9$ a 9,9$)$ & 9,1 & $(2,6 \text { a } 16,0)^{\star \star}$ \\
\hline \multicolumn{8}{|l|}{4 a 15 días } \\
\hline$<25$ años & 22,5 & 9,7 & 12,5 & $-19,8$ & $(-21,7 a-17,7)^{\star \star}$ & 6,3 & $(3,8 \text { a } 8,8)^{\star \star}$ \\
\hline 25-34 años & 16,9 & 10,0 & 11,1 & $-11,8$ & $(-15,9 a-7,5)^{\star \star}$ & 2,0 & $(-1,3$ a 5,4$)$ \\
\hline 35-44 años & 14,1 & 9,5 & 10,9 & $-9,2$ & $(-13,6 a-4,4)$ & 3,2 & $(0,0 \text { a } 6,6)^{\star \star}$ \\
\hline 45-54 años & 12,8 & 8,6 & 9,6 & $-9,0$ & $(-13,7 a-4,1)^{\star \star}$ & $-2,5$ & $(-8,7$ a 4,1$)$ \\
\hline$\geq 55$ años & 11,6 & 8,9 & 9,8 & $-6,1$ & $(-11,3 a-0,5)$ & 2,0 & $(-2,0$ a 6,2$)$ \\
\hline \multicolumn{8}{|l|}{16 a 30 días } \\
\hline$<25$ años & 3,8 & 2,3 & 3,0 & $-12,7$ & $(-14,7 a-10,6)^{\star \star}$ & 5,7 & $(3,5 \text { a } 7,9)^{\star \star}$ \\
\hline 25-34 años & 3,8 & 2,7 & 3,1 & $-9,1$ & $(-17,2 a-0,1)^{\star \star}$ & 3,0 & $(-3,3$ a 9,7$)$ \\
\hline 35-44 años & 3,7 & 2,6 & 3,3 & $-8,0$ & $(-15,7 \text { a } 0,4)^{\star}$ & 4,6 & $(-1,5$ a 11,1$)$ \\
\hline 45-54 años & 4,1 & 2,7 & 3,4 & $-10,0$ & $(-14,5 a-5,2)^{\star \star \star}$ & 4,2 & $(0,5 \text { a } 8,2)^{\star \star}$ \\
\hline$\geq 55$ años & 4,8 & 3,4 & 4,1 & $-7,6$ & $(-12,0 \text { a }-3,0)^{\star \star \star}$ & 3,1 & $(-0,8$ a 7,2$)$ \\
\hline \multicolumn{8}{|l|}{31 a 90 días } \\
\hline$<25$ años & 3,9 & 2,6 & 3,0 & $-10,9$ & $(-12,6 a-9,2)^{\star \star \star}$ & 2,9 & $(1,1 \text { a } 4,8)^{\star \star}$ \\
\hline 25-34 años & 4,5 & 3,6 & 4,0 & $-6,0$ & $(-17,5$ a 7,2$)$ & 2,0 & $(-6,8$ a 11,7$)$ \\
\hline 35-44 años & 4,0 & 3,4 & 4,5 & $-4,8$ & $(-14,9$ a 6,5$)$ & 5,6 & $(-2,7$ a 14,6$)$ \\
\hline 45-54 años & 4,5 & 3,7 & 4,5 & $-4,9$ & $(-11,3$ a 1,9$)$ & 3,7 & $(-0,9$ a 8,5$)$ \\
\hline$\geq 55$ años & 6,5 & 5,2 & 6,1 & $-5,4$ & $(-9,1 a-1,6)^{\star \star}$ & 3,9 & $(0,0 \text { a } 7,9)^{\star}$ \\
\hline \multicolumn{8}{|l|}{ > 90 días } \\
\hline$<25$ años & 2,2 & 1,1 & 1,2 & $-17,0$ & $(-22,6 a-10,9)^{\star \star}$ & 1,1 & $(-5,4$ a 8,1$)$ \\
\hline 25-34 años & 2,5 & 1,6 & 2,3 & $-10,3$ & $(-21,9$ a 3,0$)$ & 5,4 & $(-5,2$ a 17,2$)$ \\
\hline 35-44 años & 2,7 & 1,7 & 2,8 & $-10,8$ & $(-20,1 a-0,6)^{\star \star}$ & 9,5 & $(0,3 \text { a } 19,4)^{\star \star}$ \\
\hline 45-54 años & 3,7 & 2,5 & 3,6 & $-9,4$ & $(-15,9 a-2,5)^{\star \star}$ & 6,6 & $(1,0 \text { a } 12,5)^{\star \star}$ \\
\hline$\geq 55$ años & 5,8 & 4,0 & 6,0 & $-8,5$ & $(-13,3 a-3,5)^{\star \star}$ & 7,2 & $(2,5 \text { a 12,1 })^{\star \star}$ \\
\hline \multicolumn{8}{|l|}{ Global } \\
\hline$<25$ años & 47,8 & 25,9 & 42,6 & $-16,0$ & $(-17,9 a-14,0)^{\star \star}$ & 11,4 & $(8,2 \text { a } 14,7)^{\star \star}$ \\
\hline 25-34 años & 38,5 & 27,7 & 37,8 & $-8,5$ & $(-13,6 a-3,0)^{\star \star}$ & 6,2 & $(1,9 \text { a } 10,7)^{\star \star}$ \\
\hline 35-44 años & 32,0 & 24,5 & 33,9 & $-6,9$ & $(-12,4 a-1,2)^{\star \star}$ & 6,9 & $(2,4 \text { a 11,7) })^{\star \star}$ \\
\hline 45-54 años & 30,7 & 22,6 & 29,5 & $-7,6$ & $(-12,9 a-2,1)^{\star \star \star}$ & 5,4 & $(1,3 \text { a } 9,7)^{\star \star \star}$ \\
\hline$\geq 55$ años & 32,7 & 25,8 & 32,4 & $-5,7$ & $(-10,6 a-0,5)^{\star \star}$ & 4,7 & $(0,2 \text { a } 9,3)^{\star \star}$ \\
\hline
\end{tabular}

${ }^{*} p<0,10 ;{ }^{* *} p<0,05$. 
Tabla 3: Incidencia de incapacidad temporal de origen común (ITCC) con sus Intervalo de Confianza del 95\% (IC 95\%) y porcentaje de cambio anual (PCA) por tramos de duración según sexo durante los períodos 2009-2013 y 2013-2018.

\begin{tabular}{|c|c|c|c|c|c|c|c|}
\hline & & & & & 2009-2013 & & 2013-2018 \\
\hline & $\begin{array}{l}\text { Tasa } \\
2009\end{array}$ & $\begin{array}{l}\text { Tasa } \\
2013\end{array}$ & $\begin{array}{l}\text { Tasa } \\
2018\end{array}$ & PCA & (IC 95\%) & PCA & (IC 95\%) \\
\hline \multicolumn{8}{|c|}{1 a 3 días } \\
\hline Mujer & 9,4 & 8,0 & 13,9 & $-6,2$ & $(-12,1 \text { a } 0,1)^{\star}$ & 12,2 & $(5,9 \text { a } 19,0)^{\star \star}$ \\
\hline Hombre & 8,0 & 6,6 & 11,2 & $-6,1$ & $(-13,9 \text { a } 2,3)^{\star \star \star}$ & 11,3 & $(3,7 \text { a } 19,3)^{\star \star \star}$ \\
\hline \multicolumn{8}{|c|}{4 a 15 días } \\
\hline Mujer & 17,3 & 10,5 & 11,5 & $-11,3$ & $(-13,9 a-8,7)^{\star \star \star}$ & 2,0 & $(0,4 \text { a } 3,6)^{\star \star \star}$ \\
\hline Hombre & 13,6 & 8,3 & 9,7 & $-11,5$ & $(-14,7 a-8,1)^{\star \star}$ & 1,9 & $(5,5 \text { a } 3,5)^{\star \star}$ \\
\hline \multicolumn{8}{|c|}{16 a 30 días } \\
\hline Mujer & 4,7 & 3,3 & 3,9 & $-9,4$ & $(-10,8 a-7,9)^{\star \star}$ & 3,4 & $(1,9 \text { a } 4,9)^{\star \star}$ \\
\hline Hombre & 3,3 & 2,3 & 2,9 & $-8,9$ & $(-11,8 a-6,0)^{\star \star}$ & 5,1 & $(2,9 \text { a } 7,4)^{\star \star}$ \\
\hline \multicolumn{8}{|c|}{31 a 90 días } \\
\hline Mujer & 5,7 & 4,5 & 5,4 & $-5,7$ & $(-8,0 a-3,3)^{\star \star}$ & 3,2 & $(1,0 \text { a } 5,4)^{\star \star}$ \\
\hline Hombre & 3,6 & 2,9 & 3,7 & $-5,9$ & $(-10,9 a-0,7)^{\star \star}$ & 5,6 & $(1,8 \text { a } 9,6)^{\star \star}$ \\
\hline \multicolumn{8}{|c|}{ > 90 días } \\
\hline Mujer & 3,9 & 2,5 & 3,9 & $-10,2$ & $(-15,1 \text { a }-4,9)^{\star \star \star}$ & 8,2 & $(2,8 \text { a } 14,0)^{\star \star *}$ \\
\hline Hombre & 2,4 & 1,7 & 2,5 & $-9,1$ & $(-17,7 \text { a } 0,3)^{\star}$ & 7,6 & $(-0,7 \text { a } 16,7)^{\star}$ \\
\hline \multicolumn{8}{|l|}{ Global } \\
\hline Mujer & 41,0 & 28,8 & 38,5 & $-8,9$ & $(-11,1 a-6,7)^{\star \star}$ & 6,0 & $(4,0 \text { a } 8,1)^{\star \star}$ \\
\hline Hombre & 30,9 & 21,9 & 30,1 & $-8,9$ & $(-11,6 a-6,1)^{\star \star}$ & 6,9 & $(4,8 \text { a } 9,1)^{\star \star}$ \\
\hline
\end{tabular}

${ }^{*} p<0,10 ;{ }^{* *} p<0,05$.

\section{Discusión}

Los resultados muestran un patrón consistente de descenso, y posterior ascenso con un punto de inflexión en 2013, coincidiendo con lo reportado en diferentes informes oficiales ${ }^{(1,17,18)}$. Al mismo tiempo, el estudio muestra que la incidencia global sin segmentar está determinada fundamentalmente por los procesos de corta duración (menor de 16 días).

La caída de la incidencia observada durante el período 2009-2018, coincide con el periodo de crisis económica, donde se produjo un aumento en la tasa de desempleo ${ }^{(19)}$. Este descenso observado en todos los tramos de duración, pero más destacado en los de 4 a 15 días y más de 90 días, en los trabajadores más jóvenes, podría explicarse en parte, por el efecto disciplina, que ante un fenómeno de crisis las personas prescindan de las bajas cortas al percibir que la situación laboral es 
más inestable(19-21). Este mismo efecto disciplina, junto con el efecto de selección, aumentan la probabilidad de expulsar del empleo a los trabajadores con peor salud, lo que podría favorecer un descenso en las bajas largas ${ }^{(19-21)}$.

También el descenso de la incidencia más acusado en los jóvenes, se produce simultáneamente con la reducción progresiva de dicho colectivo dentro de la población estudiada (datos no mostrados), al ser uno de los grupos de edad más afectados durante la crisis económica.

El cambio evolutivo en la tasa de incidencia a partir del 2013, con un ascenso que se mantiene hasta la fecha, en parte es atribuido a una mejora del ciclo económi$\mathrm{co}$. Al reducirse el desempleo se han vuelto a recuperar las tasas de incapacidad temporal que existían antes de la crisis ${ }^{(19)}$. Ahora bien, vale la pena plantearse si hay realmente una mayor frecuencia de procesos, o es bastante similar a momentos anteriores. Tal como describe Castejon, los valores actuales no son los más altos, y España no encabeza el absentismo laboral a nivel Europeo(22). Sin embargo, lo que sí existe actualmente es una situación coyuntural que dificulta la gestión (menos recursos económicos para la gestión por parte de las mutuas, aumento en las propuestas de altas rechazadas, entre otros) ${ }^{(2)}$. De forma complementaria, es posible que el ascenso de la incidencia de las contingencias comunes a expensas de procesos muy cortos (1 a 3 días), coincida con la puesta en marcha del Real Decreto 625/2014, ya que permite que los médicos de la seguridad social puedan emitir el parte de baja y alta en un mismo acto(23). Este hecho puede haber favorecido el alargamiento de los procesos de muy corta duración.

Respecto al patrón ascendente de la incidencia en el tramo de más de 90 días, especialmente en los mayores de 55 años, es posible que sea el resultado de la confluencia del cambio de ciclo económico que favorece la incorporación de trabajadores con peor estado de salud, junto con un envejecimiento de la plantilla( ${ }^{(24)}$.

De cara la gestión de estas bajas extremas, nuestra hipótesis es que las bajas cortas y largas son fenómenos diferentes, en cuanto obedecen a causas distintas, y por lo tanto requieren diferentes abordajes. Este abordaje está más asentado en otros lugares como el Reino Unido, donde desde las organizaciones separan la gestión de dichos procesos ${ }^{(25-27)}$. Dada la incapacidad de las mutuas de intervenir efectivamente en los procesos antes del día 15, la gestión de la baja corta debería estar orientada hacia su prevención desde la empresa. Ello requiere la implicación de toda la organización, empezando desde la dirección general. Fundamentalmente se traslada el eje de la gestión de recursos humanos al mando, ya que es quien mejor conoce a los trabajadores de su equipo. El ascenso de la ITCC de corta duración más pronunciado en los jóvenes respecto a los otros grupos de edad, podría evidenciar la necesidad de intervenciones para mejorar el equilibrio personal-laboral, el compromiso y la satisfacción con el trabajo ${ }^{(3)}$. En la gestión de las bajas largas, donde se observa el ascenso más pronunciado en la población trabajadora de mayor edad, hay una participación tanto de las mutuas, como de las empresas. En éstos casos lo que se pretende es reducir la duración de los procesos y facilitar una pronta y adecuada reincorporación al trabajo en los casos que sea posible(3). 
Una limitación del estudio es que realiza una descripción de la incidencia por contingencia común sin considerar otros factores de interés. Sabemos que detrás de dichos valores existen comportamientos heterogéneos por actividad económica, ya que la crisis no afectó a todos los sectores por igual, ni en el mismo momento. A pesar de dicha limitación consideramos que los resultados mostrados son de gran utilidad para aquellos profesionales que se dedican a la prevención y gestión de la ITCC. Por otro lado, los resultados obtenidos están basados en los datos de una única mutua. Sin embargo, creemos que los patrones observados en la tendencia temporal entre los diferentes grupos de duración de la ITCC serán similares al conjunto del estado español, debido a: 1) los indicadores globales de la mutua coinciden con las estadísticas de la Seguridad Social, 2) la mutua presenta una amplia cobertura geográfica y tamaño de la población protegida.

Para valorar con más detalle la existencia de puntos de inflexión en la tendencia se ha optado por realizar una regresión LOESS. Cabe destacar que se pueden aplicar otros métodos posibles con este fin ${ }^{(13,28-29)}$. Consideramos que la inspección visual es una manera sencilla de mostrar el cambio en la tendencia, incluso para audiencias no especializadas. Como dijo John Tukey: "Cuando se comunican los resultados a no técnicos, no hay nada mejor que una visualización clara para mostrar tu afirmación"(30).

En resumen, el análisis global no habría permitido identificar los tramos de duración con mayor descenso en la incidencia (4-15 y 90 días, principalmente en jóvenes) y los de mayor ascenso (1-3 días en jóvenes y más de 90 días en mayores). Por consiguiente, la descripción temporal de la incidencia de ITCC por tramos de duración, segmentado por factores como edad y sexo, muestra una visión más detallada del comportamiento de la incapacidad temporal, permite plantear intervenciones más específicas en la gestión de la ITCC, así como evaluar temporalmente dichas intervenciones.

\section{Bibliografía}

1. Estadísticas, presupuestos y estudios. Incapacidad Temporal. Ministerio de Trabajo, Migraciones y Seguridad Social [sede web]. [citado 8 Jul 2019]. Disponible en: http://www.seg-social.es/wps/portal/wss/internet/EstadisticasPresupuestosEstudios/Estadisticas/EST45/EST46

2. Asociación de Mutuas de Accidentes de Trabajo AMAT [sede web]. VII Informe Adecco sobre absentismo. 2019. [citado 8 Jul 2019]. Disponible en: https://www. amat.es/

3. Sampere M, López JC, Ballesteros M, Sacristán R, Plana M, Alguacil S y col. Segmentación de la incidencia de la incapacidad temporal según su duración como estrategia de análisis. Arch Prev Riesgos Labor 2018;21:203-205.

4. Duran X, Martínez JM, Benavides FG. Tendencia temporal de la incapacidad laboral permanente en España (1992-2010). Rev Esp Salud Publica. 2012;86:533-42. 
5. Väänänen $A$, Toppinen-Tanner $S$, Kalimo R, Mutanen P, Vahtera J, Peiró JM. Job characteristics, physical and psychological symptoms, and social support as antecedents of sickness absence among men and women in the private industrial sector. Social Science \& Medicine. 2003;57: 807-824.

6. Hensing G, Alexanderson $\mathrm{K}$ The association between sex segregation, working conditions, and sickness absence among employed women. Occup Environ Med 2004;61:e7. [edición electrónica]. [citado 8 Jul 2019]. Disponible en: https://oem. bmj.com/content/oemed/61/2/e7.full.pdf

7. Jansen N, Kant IJ, van Amelsvoort L, Kristensen, Swaen G, Nijhuis F. Work-family conflict a risk factor for sickness absence. Occup Environ Med 2006;63:488494.

8. Taimela S, Läärä E, Malmivaara A, Tiekso J, Sintonen H, Juste'n S, Aro T. Self-reported health problems and sickness absence in different age groups predominantly engaged in physical work. Occup Environ Med. 2007;64:739-746.

9. Sampere M, Ballesteros M, López JC. La edad y el género influyen en la incapacidad temporal por contingencia común. [edición electrónica]. [citado 8 Jul 2019]. Disponible en: https://mejoratuabsentismo.mc-mutual.com/-/la-edad-y-el-genero-influyen-en-la-incapacidad-temporal-por-contingencia-com-1

10. Cleveland W. Robust Locally Weighted Regression and Smoothing Scatterplots. Journal of the American Statistical Association 1979;74:829-836.

11. Golub, G, Heath, M, Wahba, G. Generalized cross validation as a method for choosing a good ridge parameter. Technometrics. 1979;21:215--223.

12. Wang XF (2010). fANCOVA: Nonparametric Analysis of Covariance. R package version 0.5-1 [Programa Informático]. [citado 8 Jul 2019]. Disponible en: https:// cran.r-project.org/web/packages/fANCOVA/

13. Kim HJ, Fay MP, Feuer EJ, Midthune DN. Permutation tests for joinpoint regression with applications to cancer rates. Stat Med. 2000;19:335-51.

14. R Core Team (2018). R: A language and environment for statistical computing. [Programa Informático]. R Foundation for Statistical Computing, Vienna, Austria. [citado 8 Jul 2019]. Disponible en: http://www.R-project.org/

15. RStudio Team (2018). RStudio: Integrated Development for R. [Programa Informático]. RStudio, Inc., Boston, MA. [citado 8 Jul 2019]. Disponible en: http://www. rstudio.com/

16. Reglamento (UE) 2016/679 del Parlamento Europeo y del Consejo, de 27 de abril de 2016, relativo a la protección de las personas físicas en lo que respecta al tratamiento de datos personales y a la libre circulación de estos datos y por el que se deroga la Directiva 95/46/CE (Reglamento general de protección de datos). Agencia Estatal Boletín Oficial del Estado. [sede web]. [citado 8 Jul 2019]. Disponible en: https://www.boe.es/buscar/doc.php?id=DOUE-L-2016-80807

17. La Incapacidad Temporal por Contingencias Comunes (Enfermedad Común y Accidente no laboral) en Navarra. Año 2017. [edición electróni- 
ca]. [citado 8 Jul 2019]. Disponible en: http://www.navarra.es/NR/rdonlyres/ 87FA3162-CB6D-41EF-8ACE-48CA0A40F9DF/426146/INFORMEIT2017.pdf

18. Checa J. Capítulo 5. Marco de Gestión I. Estudio de la evolución de la Incapacidad Temporal 2009-2018. VIII Informe Adecco Sobre Absentismo. [Informe monográfico]. [citado 8 Jul 2019]. Disponible en: https://www.amat.es/Ficheros/17969. pdf

19. López JC, Ballesteros M, Sampere M, Sacristán R, Alguacil S, Ruiz A y col. Relación entre las tasas de desempleo y los indicadores de absentismo por incapacidad temporal durante periodos de crisis económica. Arch Prev Riesgos Labor. 2018:21:80-84.

20. Dyrstad JM, Ose SO. Non-linear Unemployment Effects in Sickness Absence: Discipline or Composition Effects? Department of Economics. Norwegian University of Science and Technology. Working Paper No 25. 2002. [citado 8 Jul 2019]. Disponible en: https://brage.bibsys.no/xmlui/handle/11250/267159

21. Arai $M$, Thoursie PS. Incentives and selection in cyclical absenteeism. Labour Econ. 2005; 12:269-280.

22. Castejón E. A vueltas con el absentismo: ¿un problema grave u obsesión paranoide? [sede web]. [citado 8 Jul 2019]. Disponible en: https://www.prevencionintegral.com/comunidad/blog/entre-sin-llamar/2018/09/25/vueltas-con-absentismo-problema-grave-u-obsesion-paranoide

23. Real Decreto 625/2014, de 18 de julio. Publicado en BOE núm. 176 de 21 de Julio de 2014. Vigencia desde 01 de Septiembre de 2014. [citado 8 Jul 2019]. Disponible en: https://www.boe.es/eli/es/rd/2014/07/18/625.

24. Ballesteros M, Sacristán R, López JC, Sampere M, Ruíz A, Santos E y col. Cambios demográficos en el ámbito laboral: envejecimiento de la población trabajadora en una empresa. Seguridad y Salud en el trabajo. 2016;57:26-34.

25. Personnel today. [sede web]. [citado 8 Jul 2019]. Disponible en: https://www. personneltoday.com/wellbeing/sickness-absence/

26. Managing Sickness Absence. Labour Relations Agency. [sede web]. [citado 8 Jul 2019]. Disponible en: https://www.Ira.org.uk/images/publications/managing_ sickness_absence_february_2013_-_3.pdf

27. CIPD. Absence Management Survey. sede web]. [citado 8 Jul 2019]. Disponible en: https://www.cipd.co.uk/knowledge/fundamentals/relations/absence/absence-management-surveys

28. Fernandez E, González JR, Borràs JM, Moreno V, Sánchez V, Peris M. Recent decline in cancer mortality in Catalonia (Spain). A joinpoint regression analysis. Eur J Cancer. 2001;37:2222-8.

29. Puig X, Ginebra J, Gispert R. Analysis of mortality time trend using generalized linear models. Gac Sanit. 2005;19:481-5.

30. Famous Quotes \& Sayings. [sede web]. [citado 8 Jul 2019]. Disponible en: http://quotetab.com/quotes/by-john-tukey\#hcYks6g05Chvskyd.97 